\title{
Epidemiologia do escorpionismo na faixa etária pediátrica no estado de Minas Gerais
}

\author{
Epidemiology of scorpionism in the pediatric age group in the State of Minas Gerais \\ Epidemiología del escorpionismo en el grupo de edad pediátrica en el estado de Minas \\ Gerais
}

Caio Augusto de Lima1 ${ }^{1}$, Caroline Coutinho Horácio Alves ${ }^{1}$, Kaio Saramago Mendonça ${ }^{1}$, Priscilla Larissa Silva Pires ${ }^{1}$, Nathalia Amado da Silva Medeiros ${ }^{1}$, Elias Rodrigues de Almeida Júnior ${ }^{1}$, Mariana Carvalho ${ }^{1}$, Tatiany Calegari ${ }^{1}$, Stefan Vilges de Oliveira ${ }^{1 *}$.

\section{RESUMO}

Objetivo: Descrever o perfil epidemiológico do escorpionismo em crianças até 14 anos no estado de Minas Gerais, Brasil. Métodos: Estudo epidemiológico, transversal, descritivo que analisou os acidentes produzidos por escorpiões em crianças, ocorridos no estado de Minas Gerais, notificados no período de janeiro de 2007 a dezembro de 2017. Resultados: Foram registrados casos de escorpionismo em 699 municípios de Minas Gerais. Os acidentes aconteceram com maior frequência na faixa etária de 10 a 14 anos, com 13.430 casos (37,63\%). No grupo de um a quatro anos ocorreram 8.236 casos (23,07\%) e no de cinco a nove anos 11.446 casos (32,07\%). As regiões corporais mais acometidas por picadas foram o pé com $8.425(23,60 \%)$ casos, dedo da mão com 7.244 $(20,30 \%)$, mão com 5.326 (14,92\%) e dedo do pé com 3.512 (9,84\%). Conclusão: No período de dez anos o perfil epidemiológico foi de crianças pardas do sexo masculino, na faixa etária de 10 a 14 anos de idade, que habitam a região urbana, com evolução para cura e sobrevida. As regiões anatômicas mais acometidas foram os dedos das mãos e os pés. As manifestações clínicas foram predominantemente de leve intensidade, sendo primordial que ocorra a busca imediata por atendimento e tratamento.

Palavras-chave: Animais venenosos, Picadas de escorpião, Epidemiologia.

\section{ABSTRACT}

Objective: Describe the epidemiological profile of scorpionism involving children up to 14 years of age in the state of Minas Gerais, Brazil. Methods: Epidemiological, cross-sectional, descriptive study that analyzes the accidents caused by scorpions in children occurred in the state of Minas Gerais, reported from January 2007 to December 2017. Results: Cases of scorpionism were registered in 699 cities in the state of Minas Gerais. Accidents occur more frequently in the 10 to 14 age group, with 13,430 cases $(37.63 \%)$. In the 1 to 4 -year-old group occurred 8,236 cases $(23.07 \%)$ and five to nine-year-old group 11,446 cases $(32.07 \%)$. The body regions most affected by bites were the foot $8,425(23.60 \%)$, finger 7,244 (20.30\%), hand $5.326(14.92 \%)$ and toe $3.512(9.84 \%)$. Conclusion: In the ten-year period, the epidemiological profile was of brown male children, aged 10 to 14 years old, who live in the urban region, with evolution to cure and survival. The most affected anatomical regions were the fingers and toes. The clinical manifestations were predominantly mild, and it is essential that there is an immediate search for care and treatment.

Keywords: Animals poisonous, Scorpion stings, Epidemiology.

\section{RESUMEN}

Objetivo: Describir el perfil epidemiológico del escorpionismo en niños hasta los 14 años en el estado de Minas Gerais, Brasil. Métodos: Estudio epidemiológico, transversal, descriptivo que analizó los accidentes causados por escorpiones en niños, ocurridos en el estado de Minas Gerais, reportados entre enero de 2007 y diciembre de 2017. Resultados: Se registraron casos de escorpionismo en 699 municipios del estado de Minas Gerais. Los accidentes ocurrieron con mayor frecuencia en el grupo de edad de 10 a 14 años, con 13.430 casos (37,63\%). En el grupo de uno a cuatro años hubo 8.236 casos $(23,07 \%)$ y en el grupo de cinco a nueve años, 11.446 casos (32,07\%). Las regiones corporales más afectadas por mordeduras son el pie con $8.425(23,60 \%)$ casos, el dedo con 7.244 $(20,30 \%)$, la mano con $5.326(14,92 \%)$ y el dedo del pie con $3.512(9,84 \%)$. Conclusión: En el período de diez años el perfil epidemiológico fue de los niños varones morenos, de 10 a 14 años, que viven en la región urbana, con evolución para la curación y supervivencia. Las regiones anatómicas más afectadas fueron los dedos de las manos y pies. Las manifestaciones clínicas fueron predominantemente de intensidad leve, siendo primordial que se produzca la búsqueda inmediata de asistencia y tratamento.

Palabras clave: Animales venenosos, Picaduras de escorpión, Epidemiología.

${ }^{1}$ Universidade Federal de Uberlândia (UFU), Uberlândia - MG. *E-mail: stefan@ufu.br 


\section{INTRODUÇÃO}

O Brasil é um país de grande diversidade geográfica, ecológica e climática, o que propicia a existência e o desenvolvimento de vários tipos de animais peçonhentos, entre eles, o escorpião (SOUZA CMV, et al., 2018). Ademais, a rápida e desordenada urbanização do território, aliada às condições de moradia humana, facilitam a rápida proliferação desses animais, o que potencializa o contato com seres humanos, resultando em acidentes com envenenamento que podem ocasionar óbito (SOUZA CMV, 2018; SILVA PM, et al., 2018). Casos de escorpionismo têm aumentado significativamente, constituindo um problema emergente de saúde pública no país, sendo mais frequentes em áreas secas, úmidas, costeiras e regiões urbanas (CUPO P, 2015; FONTENELE GC, et al., 2015; SHIBAKURA AAK, et al., 2020).

O escorpião é um artrópode quelicerado, vivíparo, que possui na extremidade de sua cauda uma estrutura denominada telson, a qual contém um par de glândulas produtoras de veneno, e um ferrão utilizado para inocular peçonha. A espécie Tityus serrulatus (escorpião amarelo) reproduz por partenogênese, ou seja, não necessita de acasalamento para a reprodução, fato que facilita a sua dispersão (BRASIL, 2009). Existem no Brasil, três espécies de escorpiões do gênero Tityus responsáveis por acidentes humanos: o $T$. serrulatus (escorpião amarelo), o T. baienses (escorpião marrom), e o T. stigmurus (escorpião do nordeste), sendo o escorpião amarelo o causador da maioria das eventualidades graves (SOUZA CMV, et al., 2018; CUPO P, 2015).

Este artrópode possui hábito noturno, adentrando em domicílios através de frestas em portas, paredes e janelas, além da rede de tubulações/encanamentos. Por não gostarem de claridade, ficam escondidos durante o dia, dentro de armários, gavetas, calçados, entre outros itens, para se proteger. São carnívoros alimentandose de insetos como baratas e grilos. Seus predadores são: aranhas, serpentes, sapos, lacraias, formigas, lagartos, aves e alguns mamíferos (SHIBAKURA AAK, et al., 2020).

De acordo com o Ministério da Saúde há algumas regiões brasileiras que possuem taxas elevadas de acidentes escorpiônicos, destacando-se o nordeste com 447.971 ocorrências, seguido pelo sudeste com 397.187. Nesta região, Minas Gerais está em primeiro lugar dentre os estados com 223.033 casos BRASIL, 2020a). Das notificações entre 2000 até 2017, o ano de 2009 sobressaiu com 49 óbitos no estado mineiro, sendo a espécie Tityus serrulatus uma das principais responsáveis, uma vez que é considerada a mais perigosa, pois sua picada pode ser letal, principalmente quando se trata de crianças e pessoas debilitadas (BRASIL, 2020a; BRASIL, 2020b).

A notificação ao Ministério da Saúde de acidente envolvendo animal peçonhento é obrigatória, para que se reconheça o perfil epidemiológico e consequentemente ocorram ações de vigilância, assistência em saúde e a disponibilização de antivenenos específicos levando em consideração o animal agressor (FONTENELE GC, et al., 2015).

Os sintomas apresentados após a picada de um escorpião são classificados em leves, moderados ou graves. A severidade depende não apenas da dimensão e da espécie escorpiônica, mas também da quantidade de veneno inoculado, da massa corpórea da vítima e a sua sensibilidade em relação à substância tóxica injetada (BRASIL, 2001).

As pessoas mais expostas a estes artrópodes são crianças; trabalhadores de construção civil; donas de casa e os que trabalham com madeireiras, transportadoras e distribuidoras de hortifrutigranjeiros, visto que, o escorpião pode estar escondido entre estes itens (SHIBAKURA AAK, et al., 2020).

Os acidentes na faixa etária pediátrica têm como manifestações sistêmicas: vômitos, dor abdominal, diarréia, arritmias cardíacas, taquipneia, confusão mental, hipotensão e hipertensão arterial, sonolência e até mesmo a sudorese profunda, que são considerados sintomas graves e devem ser encaminhados imediatamente ao serviço de saúde para que possam receber a soroterapia o mais rápido possível (BRASIL, 2001; SHIBAKURA AAK, et al., 2020).

É consenso que os acidentes durante a infância são questões de saúde pública, pois podem causar sequelas permanentes ou até mesmo o óbito. Situações graves que requerem hospitalização infantil potencializam condições emocionais, como o estresse e trauma, já que nesse período há distanciamento do 
meio social, das atividades e hábitos próprios desta fase da vida (SIMAS VF e SOUZA AS, 2019). Pelo escorpionismo ser comum na infância é imprescindível realizar a avaliação precoce do envenenamento e estabelecer o tratamento de imediato (CHIPPAUX JP e GOYFFON M, 2008).

Diante do exposto, o presente estudo visa descrever o perfil epidemiológico pediátrico de acidentes oriundos do escorpionismo ocorridos no estado de Minas Gerais no período de janeiro de 2007 a dezembro de 2017, a partir da base de dados de acidentes por animais peçonhentos notificados no Sistema de Informação de Agravos de Notificação (SINAN).

\section{MÉTODOS}

Este trabalho consiste em um estudo descritivo, transversal, com abordagem quantitativa de casos de escorpionismo na população pediátrica notificados pelas Secretarias Municipais de Saúde dos municípios de Minas Gerais, Brasil. Os registros foram acessados por meio do SINAN durante o período de um de janeiro de 2007 a trinta e um de dezembro de 2017 provenientes da base dos dados epidemiológicos fornecida pelo Ministério da Saúde segundo o protocolo do "Sistema Eletrônico do Serviço de Informação ao Cidadão" de número 25820006595201811.

O uso do SINAN contribui para a coletivização da informação, o que permite aos profissionais da saúde acesso ao conteúdo disponibilizado, possibilitando a realização do diagnóstico dinâmico de eventualidades em uma população, viabilizando a indicação dos possíveis riscos aos quais as pessoas estão sujeitas e o provimento de subsídios para explicações causais dos agravos de notificação compulsória (SINAN, 2020).

Para realização deste estudo, utilizou-se as variáveis que contemplam a ficha de notificação e investigação dos acidentes por animais peçonhentos do SINAN. Foram selecionados os acidentes produzidos por escorpiões em crianças de zero a 14 anos de idade que reportaram as seguintes informações: faixa etária (categorizada); raça/cor do paciente (preta; parda; amarela; indígena; branca; ignorada); sexo (feminino; masculino; ignorado); zona (periurbana; urbana, rural, ignorado); data do acidente; data dos primeiros sintomas; classificação do acidente (grave; moderado; leve); local da picada (cabeça; braço; antebraço; mão; dedo da mão; tronco; coxa; perna; pé; dedo do pé; ignorado); tempo decorrido da picada ao atendimento em horas ( 0 a 1h; 1 a 3h; 3 a 6h; 6 a 12h; 12 a 24h; mais de 24h; ignorado); se apresentou manifestações locais (ignorado; não; sim); especificação se manifestações locais (necrose; equimose; edema; dor; outras); evolução do caso (cura; óbito por acidentes por animais peçonhentos; óbito por outras causas; ignorado). Ressalta-se que um mesmo paciente pode apresentar mais de um sintoma clínico, aumentando assim a frequência de cada tipo de manifestação clínica.

A estatística descritiva foi utilizada para a análise das variáveis e os resultados apresentados em medidas de frequência absoluta e relativa. Foi calculado o coeficiente de incidência dos acidentes produzidos por escorpiões em Minas Gerais a partir da fórmula: o número de acidentes no município dividido pela população e multiplicado por 100 mil habitantes, conforme as informações das estimativas populacionais projetadas pelo Instituto Brasileiro de Geografia e Estatística (IBGE, 2020). Os Softwares Excel e Tabwin 3.32 foram empregados para o desenvolvimento das análises.

Ressalta-se ainda, que a pesquisa seguiu os preceitos éticos, foi realizada a partir de um banco de dados secundários, no qual não é possível acessar qualquer informação que possibilite a identificação dos pacientes. Por este tipo de desenho de estudo, não foi requerida a submissão ao Comitê de Ética em Pesquisa com Seres Humanos (CEP), contudo foram obedecidas as recomendações da Resolução do Conselho Nacional de Saúde no 510, de 7 de abril de 2016 (BRASIL, 2016).

\section{RESULTADOS}

Foram reportadas 35.693 notificações de escorpionismo em Minas Gerais de janeiro de 2007 a dezembro de 2017 de pacientes atendidos na faixa etária pediátrica (zero até 14 anos). Houve aumento progressivo do número das notificações ao longo dos anos estudados. Neste período, foram registrados 183 óbitos e a incidência média do escorpionismo foi de 70,56 casos por 100 mil habitantes. O sexo masculino foi o predominante em $53 \%$ dos casos. O maior número de acidentes ocorreu nos meses quentes e úmidos, sendo a maioria em setembro, outubro, novembro e dezembro (Figura 1). 
Figura 1 - Número de casos, incidência e número de óbitos por escorpionismo na faixa etária pediátrica (zero a 14 anos). Minas Gerais, 2007-2017.

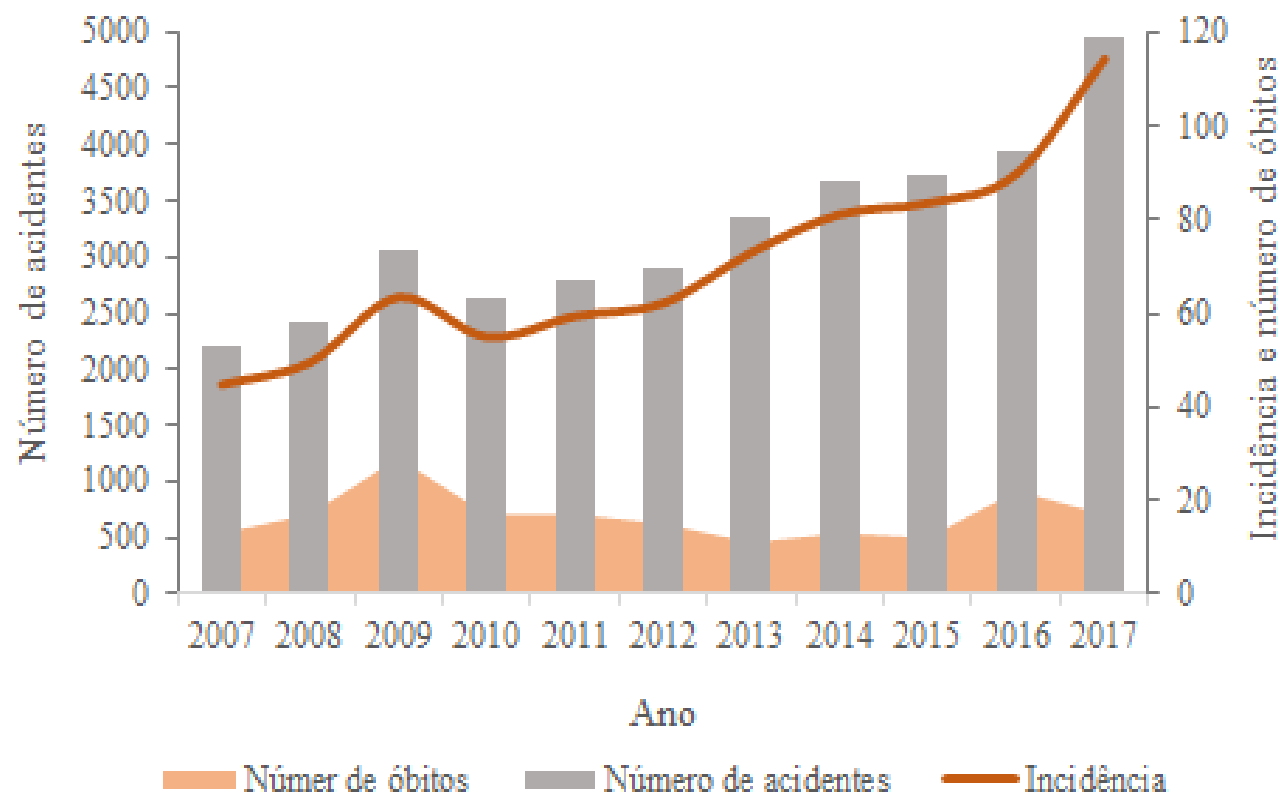

Fonte: Lima CA, et al., 2021. Dados extraídos de Sistema de Informação de Agravos de Notificação (SINAN), 2007-2017.

Foram registrados casos de escorpionismo em 699 municípios do estado de Minas Gerais, variando de um caso (mínimo) por município a 1.689 casos (máximo) por município. Os óbitos foram registrados em 115 municípios do estado, variando de um óbito (mínimo) a cinco óbitos (máximo) por município (Figura 2).

Figura 2 - A. Distribuição espacial dos casos de escorpionismo segundo o município de ocorrência do acidente. B. Distribuição espacial dos óbitos por escorpionismo segundo o município de ocorrência do acidente. Minas Gerais, 2007-2017.
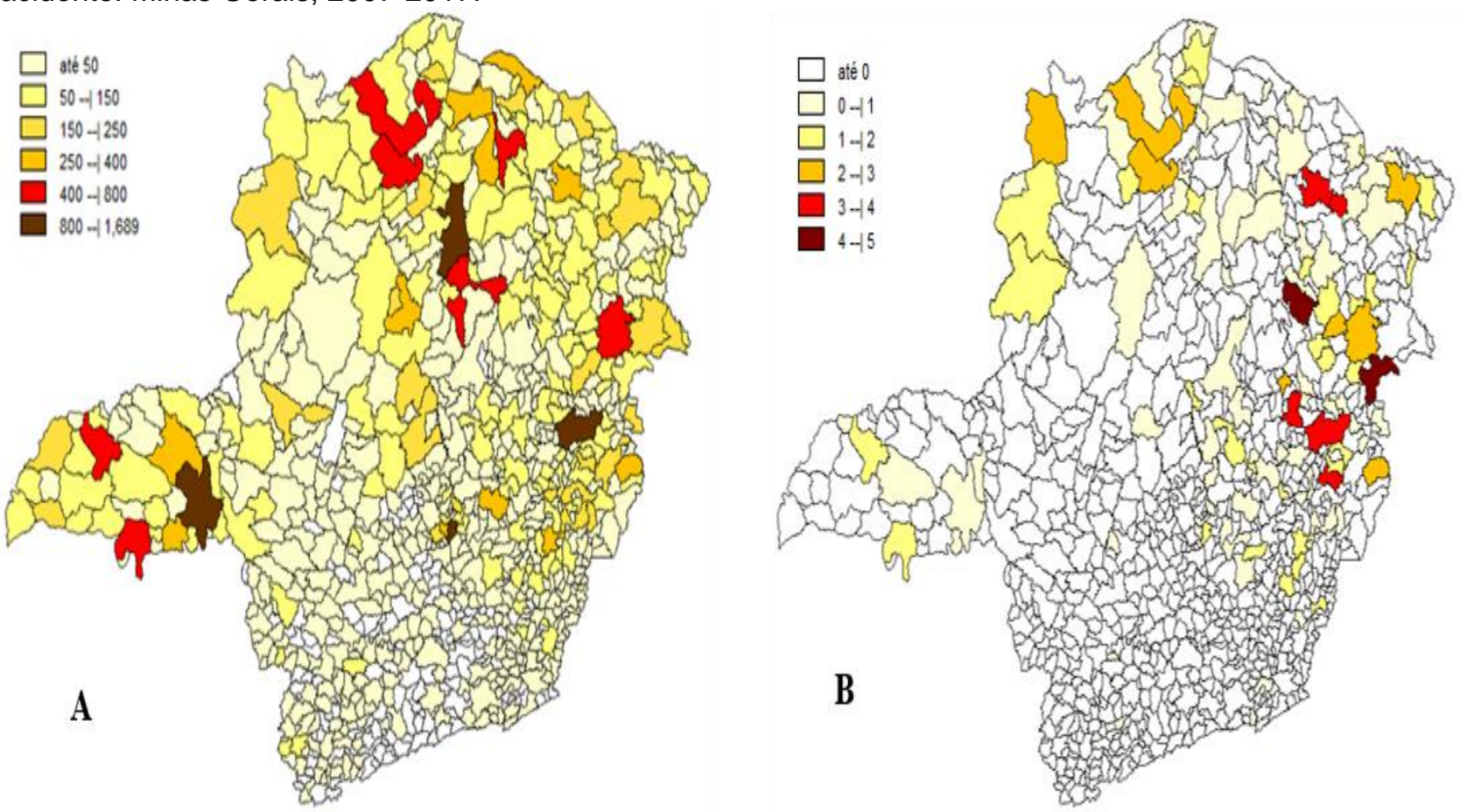

Fonte: Lima CA, et al., 2021. Dados extraídos de Sistema de Informação de Agravos de Notificação (SINAN), 2007-2017. 
Em relação ao local de ocorrência do acidente e a frequência de evolução os dados estão demonstrados a seguir (Tabela $\mathbf{1}$ ).

Tabela 1 - Caracterização dos casos, óbitos e respectivas frequências do escorpionismo na faixa etária pediátrica segundo a zona de ocorrência do acidente. Minas Gerais, 2007-2017.

\begin{tabular}{lcccc}
\hline Local de ocorrência & Número de casos & Frequência (\%) & Número de óbitos & Frequência (\%) \\
\hline Ignorado / Branco & 609 & 1,71 & 2 & 1,09 \\
Urbana & 20.380 & 57,10 & 64 & 34,97 \\
Rural & 14.407 & 40,36 & 117 & 63,93 \\
Periurbana & 297 & 0,83 & 0 & 0 \\
\hline Total & $\mathbf{3 5 . 6 9 3}$ & $\mathbf{1 0 0}$ & $\mathbf{1 8 3}$ & $\mathbf{1 0 0}$ \\
\hline
\end{tabular}

Fonte: Lima CA, et al., 2021. Dados extraídos de Sistema de Informação de Agravos de Notificação (SINAN), 2007-2017.

A frequência dos acidentes de acordo com a faixa etária e a condição étnico-racial, o intervalo entre o tempo de picada e atendimento e o desfecho final dos pacientes pediátricos são descritos na Tabela 2.

Tabela 2 - Caracterização de acidentes na infância de acordo com a característica étnico-racial, faixa etária, intervalo entre a picada e tempo de atendimento e o desfecho. Minas Gerais, 2007-2017.

\begin{tabular}{|c|c|c|}
\hline Variável & $\mathbf{N}$ & $\%$ \\
\hline \multicolumn{3}{|l|}{ Característica étnico-racial } \\
\hline Pardos & 17.990 & 50,40 \\
\hline Brancos & 9.601 & 26,90 \\
\hline Negros & 2.529 & 7,08 \\
\hline Amarelos & 441 & 1,24 \\
\hline Indígenas & 335 & 0,94 \\
\hline Ignorado/Em branco & 4.797 & 13,44 \\
\hline \multicolumn{3}{|l|}{ Idade } \\
\hline Menores de um ano & 2.581 & 7,23 \\
\hline 01-04 anos & 8.236 & 23,07 \\
\hline 05-09 anos & 11.446 & 32,07 \\
\hline 10-14 anos & 13.430 & 37,63 \\
\hline \multicolumn{3}{|c|}{ Intervalo entre a picada e o atendimento } \\
\hline Entre 0 a 1 hora & 20.800 & 58,27 \\
\hline Entre 1 e 3 horas & 10.115 & 28,34 \\
\hline Entre 3 e 6 horas & 2.150 & 6,02 \\
\hline Entre 6 a 12 horas & 484 & 1,36 \\
\hline Entre 12 a 24 horas & 317 & 0,89 \\
\hline Acima de 24 horas & 182 & 0,51 \\
\hline Ignorado/Em branco & 1.645 & 4,61 \\
\hline \multicolumn{3}{|l|}{ Desfecho } \\
\hline Pacientes curados & 34.154 & 95,7 \\
\hline Óbito pelo agravo notificado & 183 & 0,51 \\
\hline Óbito por outras causas & 14 & 0,04 \\
\hline Ignorado/Em branco & 1.342 & 3,75 \\
\hline Total & 35.693 & 100 \\
\hline
\end{tabular}

Fonte: Lima CA, et al., 2021. Dados extraídos de Sistema de Informação de Agravos de Notificação (SINAN), 2007-2017. 
As regiões anatômicas mais frequentemente atingidas são visualizadas a seguir (Figura 3), com destaque para a maior ocorrência nas extremidades dos membros superiores e inferiores.

Figura 3 - Distribuição dos acidentes na faixa etária pediátrica conforme a região anatômica do local da picada do escorpião, em Minas Gerais de 2007 a 2017.

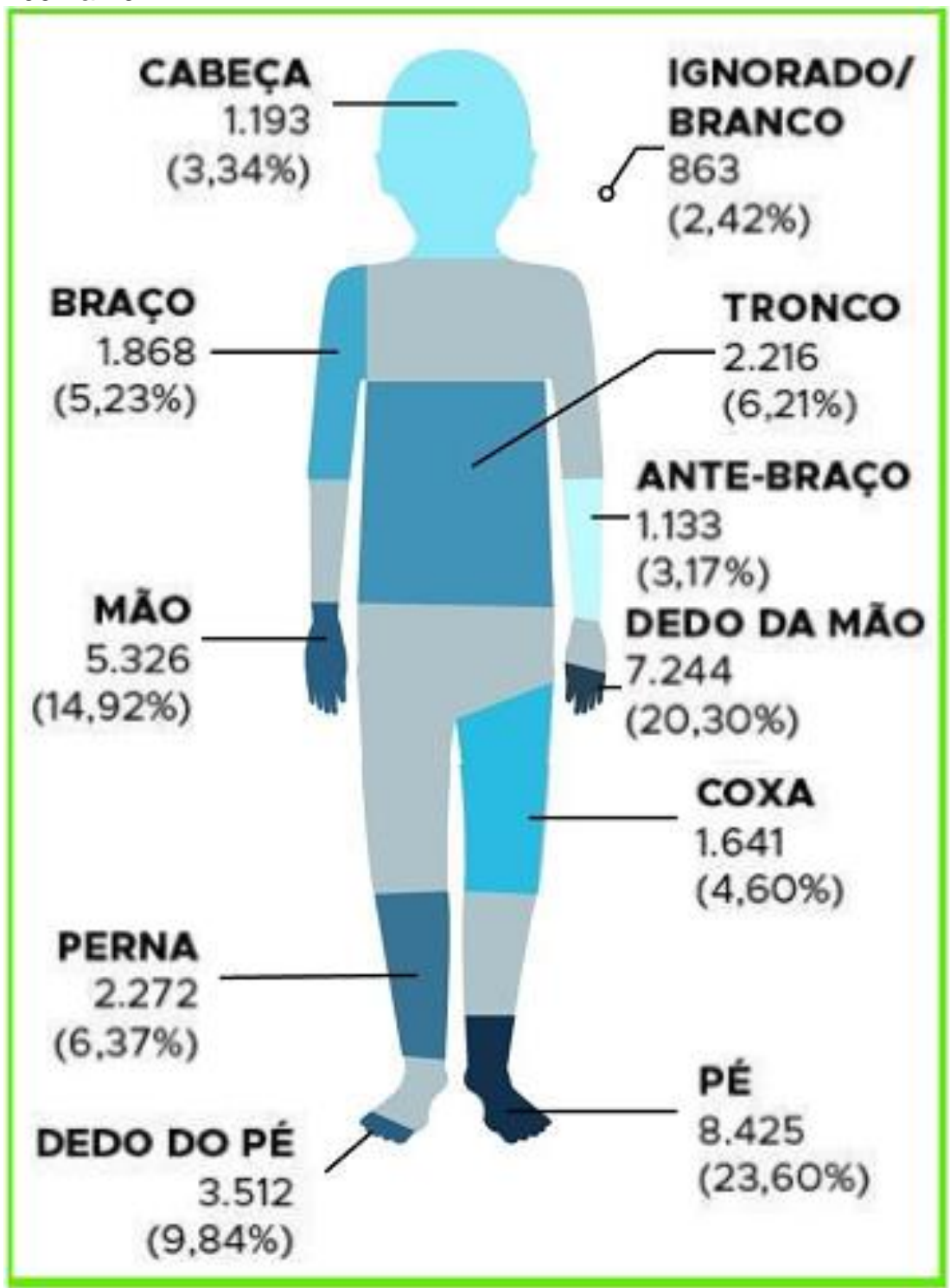

Fonte: Lima CA, et al., 2021. Dados extraídos de Sistema de Informação de Agravos de Notificação (SINAN), 2007-2017.

Após a picada foi observado que $24.699(69,20 \%)$ casos cuja manifestação clínica teve intensidade leve, $7.766(21,76 \%)$ casos com grau moderado, $2.270(6,36 \%)$ casos com exposição de sintomas graves e 958 (2,68\%) tiveram intensidade dos sinais ignorada ou em branco. Das 183 crianças que vieram a óbito pelo agravo notificado $14(7,65 \%)$ foram relacionados a sintomas leves, $26(14,81 \%)$ a sintomas moderados, 137 $(74,86 \%)$ a sintomas graves e $6(3,28 \%)$ não informaram o grau dos sinais. Outra causa foi o motivo indicado para 14 óbitos, sendo $2(14,29 \%)$ relacionados a sintomas leves, $2(14,29 \%)$ a sintomas moderados e 10 $(71,43 \%)$ a sintomas graves.

As manifestações clínicas foram definidas como local, sistêmicas e outras manifestações (Tabela 3). Em relação à conduta terapêutica $14.414(40,38 \%)$ crianças fizeram uso de soroterapia e destes pacientes foram notificados 163 (89,07\%) óbitos. 


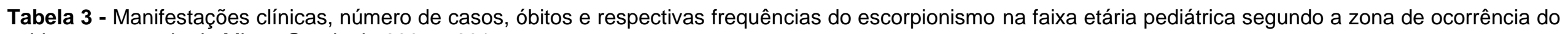
acidente no estado de Minas Gerais de 2007 a 2017.

\begin{tabular}{|c|c|c|c|c|}
\hline Variáveis & Manifestação Clínica N (\%) & Não Relatado N (\%) & Ignorado/ Branco N (\%) & Óbito pelo agravo notificado $\mathbf{N}(\%)$ \\
\hline Manifestação local & $33.266(93,20 \%)$ & $1.989(5,57 \%)$ & $438(1,21 \%)$ & $161(0,45 \%)$ \\
\hline Dor & $32.891(92,15 \%)$ & $405(1,13 \%)$ & $2.397(6,71 \%)$ & $156(0,43 \%)$ \\
\hline Edema & $11.622(32,56 \%)$ & $21.393(59,93 \%)$ & $2.678(7,50 \%)$ & $65(0,18 \%)$ \\
\hline Equimose & $786(2,20 \%)$ & $32.130(90,01 \%)$ & $2.777(7,78 \%)$ & $7(0,01 \%)$ \\
\hline Necrose & $62(0,17 \%)$ & $32.848(92,02 \%)$ & $2.783(7,79 \%)$ & $1(0,00 \%)$ \\
\hline Outras alterações locais & $2.392(6,70 \%)$ & $30.226(84,68 \%)$ & $3.075(8,61 \%)$ & $15(0,04 \%)$ \\
\hline Manifestações sistêmicas & $5.888(16,49 \%)$ & $28.684(80,36 \%)$ & $1.121(3,14 \%)$ & $145(0,40 \%)$ \\
\hline Manifestações neuroparalíticas & $307(0,86 \%)$ & $5.489(15,37 \%)$ & $29.897(83,76 \%)$ & $15(0,04 \%)$ \\
\hline Manifestações hemorrágicas & $86(0,24 \%)$ & $5.710(15,99 \%)$ & $29.897(83,76 \%)$ & $12(0,03 \%)$ \\
\hline Manifestações vagais & $4.972(13,92 \%)$ & $890(2,49 \%)$ & $29.831(83,57 \%)$ & $125(0,35 \%)$ \\
\hline Manifestações renais & $121(0,33 \%)$ & $5.659(15,85 \%)$ & $29.913(83,80 \%)$ & $24(0,06 \%)$ \\
\hline Manifestações miolíticas & $168(0,47 \%)$ & $5.611(15,72 \%)$ & $29.914(83,80 \%)$ & $11(0,03 \%)$ \\
\hline Outras manifestações sistêmicas & $1.861(5,21 \%)$ & $3.892(10,90 \%)$ & $29.940(83,88 \%)$ & $71(0,19 \%)$ \\
\hline Complicações locais & $100(0,28 \%)$ & $33.739(94,52 \%)$ & $1.854(5,19 \%)$ & $9(0,02 \%)$ \\
\hline Complicações sistêmicas & $32(0,08 \%)$ & $678(1,89 \%)$ & $632(1,77 \%)$ & $129(0,36 \%)$ \\
\hline Edema pulmonar & $480(1,34 \%)$ & $72(0,20 \%)$ & $35.141(98,45 \%)$ & $121(0,33 \%)$ \\
\hline Choque & $187(0,52 \%)$ & $346(0,96 \%)$ & $35.160(98,50 \%)$ & $64(0,17 \%)$ \\
\hline
\end{tabular}

Fonte: Lima CA, et al., 2021. Dados extraídos de Sistema de Informação de Agravos de Notificação (SINAN), $2007-2017$. 


\section{DISCUSSÃO}

Os acidentes por animais venenosos, principalmente o escorpionismo, tem se expandido em algumas regiões brasileiras, como é o caso de Minas Gerais. Nesta unidade federativa houve aumento progressivo do número de casos notificados ao SINAN no período de 2007 a 2017. No primeiro ano 2.215 casos foram informados e no último ano 4.944 casos, totalizando um aumento de aproximadamente de $122 \%$.

Na pesquisa que avaliou o escorpionismo na cidade de Belo Horizonte, Minas Gerais, entre os anos de 1990 e 1997, demonstrou-se que os acidentes produzidos por escorpiões foram mais frequentes nos meses quentes e chuvosos, com $55 \%$ dos casos, entre o período de outubro a março (SOARES MRM, et al., 2002). Em recente estudo publicado sobre os acidentes com escorpião ocorridos no leste de Minas Gerais de 2007 a 2016, os autores corroboram o período das picadas nos meses de outubro a dezembro (MATOS IM, et al., 2021). Ainda, em uma análise de casos de acidentes por animais peçonhentos no Brasil, notificados entre 2010 a 2014 pelo SINAN, verificou-se que nos dados de 2014 para todos os tipos de acidentes, inclusive os causados por escorpionismo, ocorreram com maior frequência entre os meses de outubro a abril (DOURADO FS, et al., 2014).

No tocante a sazonalidade, o maior número de acidentes representado no presente estudo ocorreu nos meses quentes e úmidos, sendo a maioria em setembro, outubro, novembro e dezembro, pois nesses períodos ocorre a reprodução dos escorpiões (SOUZA LM, et al., 2017; COSTA BC, 2011). Dados corroborados com a literatura científica revela maior frequência do escorpionismo nos períodos anuais mais chuvosos e quentes de cada região, o que no Sudeste corresponde no período entre outubro a dezembro. Um estudo retrospectivo realizado em um hospital universitário de Campinas, no estado de São Paulo, ratifica os dados encontrados neste trabalho ao evidenciar a prevalência do escorpionismo no período anual que corresponde a transição de uma estação seca e fria para uma quente e chuvosa (BUCARETCHI F, et al., 2014).

É notório que entre as regiões e estados brasileiros ocorrem divergências nos períodos de maior ocorrência de acidentes, como no estudo que avaliou o escorpionismo no estado de Goiás entre 2012 e 2013 e verificou pouca variação mensal, porém maior frequência de acidentes nos meses de abril, maio e outubro (SOUZA LM, et al., 2017) ou no estudo realizado no interior da Bahia que não evidenciou um padrão sazonal de ocorrência (CARMO EA, et al., 2016). Uma possível hipótese para tal acontecimento é que as condições ambientais locais são favoráveis durante todo o ano ou em diferentes meses, incluindo o alimento em abundância, temperatura e índices pluviométricos ideais (CARMO EA, et al., 2016).

O escorpionismo foi comprovado ser um acidente comum nas áreas urbanizadas tanto no estado de Minas Gerais quanto em Goiás, realidade explicada pela adaptação e aumento dos escorpiões as áreas urbanas, consequentemente, os episódios de escorpionismo estão relacionados ao aumento da temperatura e crescimento desordenado das cidades, somado ao desmatamento, no qual esse artrópode encontra condições de abrigo e fonte de alimento, o que permite sua reprodução com facilidade (SOUZA LM, et al., 2017; CARMO EA et al., 2016).

Com o processo de urbanização, invasão de ambientes outrora ocupados pelos escorpiões, assim como o crescimento das cidades, esses animais se adequaram às zonas urbanas em busca de abrigo, sendo eles principalmente em pilhas de tijolos, telhas e lixo. Ainda, nas áreas urbanas se alimentam especialmente de insetos, como baratas, que se proliferam em ambientes com lixos e entulhos e não possuem competidores e predadores naturais o que colabora para a acelerada proliferação desses artrópodes (FURTADO SS, et al., 2016; RECKZIEGEL GC, 2013).

A frequência do escorpionismo nas regiões urbanas pode ser favorecida e influenciada no curto intervalo de tempo entre a picada e o atendimento hospitalar, já que a maioria $(86,61 \%)$ dos casos na infância receberam atendimento médico entre 0 e 3 horas, explicando a grande porcentagem de 95,7\% dos pacientes curados e apenas $0,51 \%$ de óbitos pelo agravo notificado. No estudo de acidentes por escorpião na população geral atendida em hospital situado do leste de Minas Gerais, a maioria dos acometidos tinha até 10 anos de idade, o tempo entre a picada e 0 atendimento foi de 0 a 3 para todos os pacientes e a mortalidade observada foi expressiva na faixa etária de 0 a 14 anos de idade, sendo que os óbitos foram relacionados ao atendimento no serviço de saúde em período superior a 3 horas após a picada (MATOS IM, et al., 2021). 
Destaca-se que este tipo de acidente na zona rural frequentemente ocorre devido a suscetibilidade da população do campo à proximidade com os meios naturais e condições instáveis, sendo que em alguns casos de escorpionismo não há procura de atendimento médico, o que resulta na evolução e gravidade dos sintomas apresentados. Devido à escassez de atendimento na região rural, o sistema de saúde deveria verificar a necessidade de disponibilizar soro antiescorpiônico em locais estratégicos a fim de facilitar a demanda de atendimento e tornar mais rápido o tempo entre a picada e $o$ atendimento da população rural (CARMO EA et al., 2016).

As regiões anatômicas mais acometidas por picadas de escorpião são os membros superiores e inferiores na faixa etária pediátrica, devido às frequentes exposições das crianças a esses animais peçonhentos durante brincadeiras, das quais os entulhos e pedaços de madeira são utilizados, ou por se aventurarem ao ar livre sem o uso de calçados. Escorpiões têm hábitos domiciliares e peri domiciliares e, na maioria das vezes, escondem-se nas casas embaixo de objetos, calçados, roupas amontoadas e materiais de construção (CARMO EA et al., 2016).

Geralmente escorpiões atacam e picam apenas quando se sentem ameaçados. Nesse viés, a maioria dos acidentes envolvendo esse artrópode e humanos acomete membros superiores em virtude do manuseio de objetos em áreas normalmente úmidas e escuras, os quais servem de esconderijo e morada destes animais. Diante disso, considerando o risco da toxina produzida por escorpiões para a saúde humana, tornase necessário controlar a população destes animais, a fim de reduzir o número de acidentes e de óbitos. As medidas a serem implementadas de grande eficácia são a captura do escorpião, por meio de utensílios adequados e de proteção individual, tornar o ambiente desfavorável para a proliferação, a partir de modificações das condições do ambiente e o manejo ambiental apropriado nas áreas urbanas (BRASIL, 2009; COSTA BC, 2011).

A conscientização da população acerca da prevenção e em como proceder em caso de acidente é relevante para a diminuição de casos e agravamento do quadro clínico. A adoção de outros cuidados como afastar das paredes os berços e camas em no mínimo 10 centímetros, evitar que mosquiteiros e roupas de cama encostem no chão, examinar roupas, calçados, toalhas e tapetes antes do uso, rebocar buracos nas paredes, instalar telas em aberturas de ralos, pias e tanques, colocar tela nas janelas, vedar soleiras de portas, não acumular lixo domiciliar, limpeza de jardins, terrenos baldios, remover folhagens, conservação e limpeza de cemitérios colaboram para o controle da incidência de escorpiões e podem reduzir a ocorrência de acidentes (BRASIL, 2009; COSTA BC, 2011).

A manifestação clínica da picada de escorpião nas primeiras 24 horas pode provocar efeitos locais e/ou sistêmicos, entre os locais os mais comuns são dor, calor, edema e hematoma. Entretanto os sistêmicos variam entre hipertensão ou hipotensão arterial sistêmica, hemorragia, confusão mental, toxicidade cardiovascular, insuficiência respiratória e renal. Nesta perspectiva o risco potencial de evoluir para óbito geralmente está associado a disfunções cardíacas e respiratórias, sendo que a gravidade das manifestações clínicas da toxina escorpiônica está correlacionada a diversos fatores como idade, precocidade de início dos sintomas e a intensidade dos mesmos (CARMO EA, et al., 2019).

As crianças fazem parte do grupo mais vulnerável ao pior prognóstico, devido à relação entre a quantidade do veneno inoculado com a superfície corpórea e menor capacidade imunológica: a idade está associada com maiores níveis séricos de veneno no plasma, maior absorção do organismo, efeitos clínicos rápidos e intensos (CARMO EA, et al., 2019).

Os acidentes desencadeados por escorpionismo são classificados entre leves, moderados e graves. Acidentes leves apresentam sintomatologia local, além de agitações discretas, taquicardia e vômitos. Já os com classificação moderada apresentam manifestações sistêmicas pouco intensas como sudorese, taquipnéia, vômitos, entre outros, além dos sintomas locais. E por fim, nos acidentes graves há manifestações sistêmicas intensas como agitação psicomotora, sudorese generalizada, vômitos profusos e frequentes, tremores e hipotermia (CUPO P, et al., 2003).

As manifestações locais mais frequentes observadas no presente estudo foram dor e edema, demonstrando que após a picada, estes podem ser sinais para a procura de atendimento médico. Dentre os 
pacientes, cerca de um quinto apresentou manifestações sistêmicas nas quais as manifestações vagais foram as mais presentes nos casos de óbitos, sendo o principal sinal de complicações.

É válido destacar que os resultados obtidos foram por meio de banco de dados secundários, no qual todos os casos foram finalizados, sendo baixa a quantidade de informação denominada como ignorada. Entretanto, dados não relatados e ou ignorados, subnotificações e dados dos aspectos clínicos negligenciados podem trazer limitações a este estudo, salientando nesse sentido, a relevância dos profissionais em notificar qualquer eventualidade, pois isso retrata posteriormente a qualidade das notificações.

\section{CONCLUSÃO}

O perfil epidemiológico de acidentes pediátricos oriundos do escorpionismo em Minas Gerais no período de 2007 a 2017 foi predominante em crianças pardas do sexo masculino, na faixa etária de 10 a 14 anos de idade, com evolução para cura e sobrevida. As ocorrências na sua maioria foram na zona urbana, porém o maior número de óbitos foi relacionado à moradores da zona rural. $\mathrm{O}$ tempo entre $\mathrm{o}$ acidente $\mathrm{e} o$ atendimento no serviço de saúde foi de até uma hora. As regiões anatômicas mais acometidas nas crianças foram os pés e os dedos das mãos. As manifestações clínicas foram de leve intensidade com sintomas como dor e edema no local da picada pelo escorpião. Os acidentes escorpiônicos representam um grande risco à saúde infantil, sendo primordial que ocorra a busca imediata por atendimento e tratamento.

\section{REFERÊNCIAS}

1. BRASIL. Ministério da Saúde (BR). Casos de acidentes por escorpiões. Brasil, Grandes Regiões e Unidades Federadas, 2000 a 2018. 2020a.

2. BRASIL. Ministério da Saúde (BR). Óbitos por escorpiões. Brasil, Grandes Regiões e Unidades Federadas, 2000 a 2018. 2020b.

3. BRASIL. Ministério da Saúde (BR). Conselho Nacional de Saúde. Resolução no 510, de 7 de abril de 2016. Diário Oficial da União, 2016; 98: 44.

4. BRASIL. Ministério da Saúde (BR). Fundação Nacional de Saúde. Manual de diagnóstico e tratamento de acidentes por animais peçonhentos. 2. ed. Brasília: Fundação Nacional de Saúde, 2001; 120 p.

5. BRASIL. Ministério da Saúde (BR), Secretaria de Vigilância em Saúde. Manual de controle de escorpiões. Brasília: Ministério da Saúde, 2009; 72 p.

6. BUCARETCHI F, et al. Clinical consequences of Tityus bahiensis and Tityus serrulatus scorpion stings in the region of Campinas, southeastern Brazil. Toxicon, 2014; 89: 17-25.

7. CARMO EA, et al. Fatores associados à gravidade do envenenamento por escorpiões. Texto \& Contexto Enfermagem, 2019; 28: e20170561.

8. CARMO EA, et al. Internações hospitalares por causas externas envolvendo contato com animais em um hospital geral do interior da Bahia, 2009-2011. Epidemiologia e Serviços de Saúde, 2016; 25(1): 105-114.

9. CHIPPAUX JP, GOYFFON M. Epidemiology of scorpionism: a global appraisal. Acta Tropica, 2008; 107(2): 71-79.

10. COSTA BC. Fatores de risco para acidentes com escorpiões: uma revisão de literatura. Monografia (Especialização em Atenção Básica em Saúde da Família) - Faculdade de Medicina. Universidade Federal de Minas Gerais, Governador Valadares, 2011; $24 \mathrm{f}$.

11. CUPO P, et al. Acidentes por animais peçonhentos: escorpiões e aranhas. Medicina (Ribeirão Preto Online), 2003; 36: $490-497$.

12. CUPO P. Clinical update on scorpion envenoming. Revista da Sociedade Brasileira de Medicina Tropical, 2015; 48(6): 642-649.

13. DOURADO FS, et al. Acidentes por animais peçonhentos: análise dos dados epidemiológicos de 2014. 2014. Disponível em: http://portalarquivos.saude.gov.br/images/pdf/2016/maio/20/Informe-Epidemiol--gico-animais-pe-onhentos---.pdf. Acesso em: 10 jan. 2021.

14. FONTENELE GC, et al. Análise de intoxicações em crianças atendidas no centro de assistência toxicológica do estado do Ceará. Boletim Informativo Geum, 2015; 6(1): 33-42.

15. FURTADO SS, et al. Epidemiology of scorpion envenomation in the State of Ceará, Northeastern Brazil. Revista do Instituto de Medicina Tropical de São Paulo, 2016; 58: 15.

16. IBGE. Instituto Brasileiro de Geografia e Estatística (BR). 2020. Disponível em: https://cidades.ibge.gov.br/brasil/mg/panorama. Acesso em: 10 jan. 2021.

17. MATOS IM, et al. Scorpionism in east Minas Gerais: clinical and epidemiolical aspects. Revista Eletrônica Acervo Saúde, 2021; 13(1): e5136. 
18. SILVA PM, et al. O escorpionismo na microrregião de Goiânia, estado de Goiás (2007-2011). Revista EVS - Estudos, Vida e Saúde, 2018; 45: 55-65.

19. SIMAS VF, SOUZA AS. Perfil de crianças hospitalizadas na pediatria vítimas de acidentes na primeira infância. Revista Pró-UniverSUS, 2019; 10(1): 25-28.

20. SINAN. Sistema de Informação de Agravos de Notificação (BR). 2020 . Disponível em: https://portalsinan.saude.gov.br/. Acesso em: 10 jan. 2021.

21. SHIBAKURA AAK, et al. Notificações por picada de escorpião em um município do noroeste paulista. UNIFUNEC Ciências da Saúde e Biológicas, 2020; 3(6): 1-11.

22. SOARES MRM, et al. Escorpionismo em Belo Horizonte, MG: um estudo retrospectivo. Revista da Sociedade Brasileira de Medicina Tropical, 2002; 35(4):359-363.

23. SOUZA CMV. Escorpionismo no Brasil com ênfase no Rio de Janeiro: subsidiando políticas públicas para populações expostas. Tese (Doutorado em Informação e Comunicação em Saúde) - Instituto de Comunicação e Informação Científica e Tecnológica em Saúde. Fundação Oswaldo Cruz, Rio de Janeiro, 2018; 207 f.

24. SOUZA LM, et al. Estudo retrospectivo do escorpionismo no Estado de Goiás (2003-2012). Revista EVS - Estudos, Vida e Saúde, 2017; 44: 100-114.

25. RECKZIEGEL GC. Análise do escorpionismo no Brasil no período de 2000 a 2010. Dissertação (Mestrado em Saúde Coletiva) - Faculdade de Ciências da Saúde. Universidade de Brasília, Brasília, 2013. 103 f. 